\title{
Susceptibility to Community Associated Methicillin Resistant Staphylococcus Aureus: A Preliminary Study
}

\author{
Gan Ee Xian, ${ }^{1}$ Imam Megantara, ${ }^{2}$ Sharon Gondodiputro ${ }^{3}$ \\ ${ }^{1}$ Faculty of Medicine Universitas Padjadjaran, Indonesia, ${ }^{2}$ Department of Basic Medicine Faculty \\ of Medicine Universitas Padjadjaran, Indonesia, ${ }^{3}$ Department of Public Health Faculty of \\ Medicine Universitas Padjadjaran, Indonesia
}

\begin{abstract}
Background: The Staphylococcus aureus infection is a leading cause of infection around the world. Due to the introduction of antibiotics, a strain called Methicillin-resistant S. aureus (MRSA) has emerged and occured in hospitalized patients worldwide. However around 1990, this infection had been detected among healthy people in the community without previous health care contact, known as community associated MRSA (CA-MRSA). The objectives of this study was to identify the susceptibility to CA-MRSA among people who came to Puskesmas Jatinangor, West Java, Indonesia.

Methods: A descriptive study using purposive sampling was carried out from September to October 2014 with data obtained through anterior nasal swab of 65 patients from Puskesmas Jatinangor who fulfilled the exclusion and inclusion criteria. Samples were transported to the microbiology lab of Faculty of Medicine Universitas Padjadjaran and planted on blood agar for culturing, then identified through gram staining, catalase and coagulase tests. Lastly tested for antibiotic resistance and the zone of inhibition measuring $\leq 21 \mathrm{~mm}$ were classified as positive MRSA. The collected data were presented using tables.
\end{abstract}

Results: Out of 65 samples that were collected and tested, 17 samples (26\%) were Staphylococcus aureus (S. aureus) carriers; 6 samples (9\%) were positive with MRSA isolates; $6 / 17$ of the S. aureus are MRSA.

Conclusions: People who come to Puskesmas Jatinangor show a significantly high proportion of susceptibility to CA-MRS.

Keywords: Community associated methicillin resistant Staphylococcus aureus, community associated methicillin sensitive Staphylococcus aureus, nasal swab

\section{Introduction}

Staphylococcus aureus (S. aureus) is a wellknown type of bacteria that is a leading cause of infection worldwide. ${ }^{1}$ Hence, these bacteria are also human commensal organisms and are colonized asymptomatically in the anterior nares. ${ }^{1}$ The manifestation of Staphylococcus aureus strain infection, can range from minor skin infection to fatal pneuomonia. ${ }^{1}$

Two years after the introduction of antibiotics to treat penicillin-resistant $S$. aureus, Methicillin-resistant S. aureus (MRSA) was first reported in $1961 .^{1}$ Infection with Methicillin resistant staphylococcus aureus (MRSA) typically occurs in hospitalized patients worldwide but around 1990, this infection was detected among healthy people in a community without previous health care contact, known as community associated MRSA (CA-MRSA). ${ }^{1,2}$

Five factors of MRSA transmission have been proposed by The Centers for Disease Control and Prevention in Atlanta, known as " 5 C's". The " 5 C's" consist of Crowding, frequent skin-to-skin Contact, Compromised skin integrity, Contaminated items and surfaces, and lack of Cleanliness. ${ }^{1}$

One of the subdistricts in West Java, known as Jatinangor, is a crowded area with 4.346 people $/ \mathrm{km}^{2}$. Jatinangor is an area where many universities are located, causing huge numbers of students from different parts of the country live in this area. This condition is favourable for spreading of CA=MRSA among the people. The aim of this study was to identify the proportion of susceptibility to CA-MRSA infection in the Jatinangor community.

\section{Methods}

From September to October 2014, 65 nasal swab samples were taken by purposive sampling with 10-15 nasal swab samples per 
day, collected every Tuesday or Thursday from the Jatinangor Primary Health Care (Puskesmas) and the the laboratory testing were acrried out at the Microbiology Laboratory of Faculty of Medicine, Universitas Padjadjaran. This study was approved by the Health Research Ethics Committee Faculty of Medicine Universitas Padjadjaran.

All samples met the inclusion and exclusion criteria. The inclusion criteria included patients who visited Puskesmas Jatinangor; had no medical history of recent or current hospitalization, dialysis and surgery; had no permanent indwelling catheters or medical devices that pass through the skin into the body; had not received treatment with intranasal mupirocin or polysporin in the previous 14 days; had no treatment with oral antimicrobials for the purpose of eradicating MRSA colonization within the past 14 days (including oral rifampin therapy for any reason) and had no contraindications to nasal sampling. While the exclusion criteria were the patient who met the inclusion criteria but refused to be a respondent and also the patient who felt uncomfortable with the sample collection of the first sample swab, and refused to proceed with a second sample swab.

Nasal swabs were performed by asking the patient to attempt to clear the discharge by "blowing" his/her nose into non-scented tissue paper in one attempt. The researcher wore a mask, washed their hands, and wore clean gloves. The Amies culture swab seal were peeled open and the swab was removed. Then, the swab was inserted approximately $2 \mathrm{~cm}$ into the naris and was rotated against the anterior nasal mucosa for at least 5 times. Next, the swab was placed back into the transport tube and the end of the swab was firmly pushed to ensure that the swab wass inserted into the end of the transport tube. This was also to ensure that the swab tip was in contact with the moistened pledget, then the transport tube cap was secured. The procedure was repeated with the other naris.

The collected samples were transported to the microbiology laboratory within 8 hours and then planted directly on a blood agar plate by using the inoculation loop streak method. Cultured after 24 hours at $37^{\circ} \mathrm{C}$, colonization of bacteria was formed. The colony had a diameter about 2-3mm, round, smooth, raised and glistening, colored gray to deep golden yellow and surrounded by $\beta$-hemolysis zone that grew on the blood agar. Furthermore, for identification of S. aureus the gram staining, catalase and coagulase tests were performed.
Then, the susceptible bacteria were planted on Mueller-Hinton agar added with $30 \mu \mathrm{g}$ cefoxitim.

Gram staining was carried out by first adding a drop of normal saline on the slide then the inoculation loop was heated until red hot to make it aseptic. A small single colony was transferred from the blood agar by the inoculation loop, and then it was left to dry at room temperature. Fixation of the dried film was performed by passing it briefly through the Bunsen flame two or three times without exposing the dried film directly to the flame. Then for staining, first the slide was stained with Crystal Violet for 1 minute, washed off with distilled water then stained with iodine for 1 minute and similarly washed with distilled water. After that the slide was decolorized with $95 \%$ alcohol for $10-15$ seconds. Stain that comes out is gram negative while stain that stays is gram positive. The gram negative smear is then counterstained with safranin for 1 minute and washed with distilled water.3 Lastly, the slide was dried with filter paper and examined under a microscope with highpowered 100x objective lens with a drop of immersion oil. Staphylococcus would appear in Gram positive pair cocci, tetrads and grapelike clusters.

The catalase test was performed by allowing 1-2 drops of the 3\% hydrogen peroxide on the glass slide then an inoculation loop was used to take bacteria samples from blood agar. Presence (positive result) or absence (negative result) of bubbling or foaming was examined when the bacteria were placed on the slide.

Finally, the identification test was performed using tube coagulase test. Bacteria were inoculated by sterile method into the tube of diluted plasma, then all the tubes were incubated at $37^{\circ} \mathrm{C}$ and the suspensions were observed at half hourly intervals for a period of four hours. A positive result was indicated by gelling of the plasma, which remained in place even after inverting the tube. The test that remained negative until four hours at $37 \mathrm{oC}$ was kept at room temperature for overnight incubation.

The susceptibility test was carried out by inoculating the suspected MRSA single colonies to Muller-Hinton agar containing $30 \mu \mathrm{g}$ cefoxitin. Using the Kirby-Bauer Method, bacterial suspension was prepared in sterile aquadest with the haziness suitable according to the $0.5 \mathrm{McF}$ arland standard, which is equal to 108 Colony Forming Unit (CFU) bacteria in every one millimeter bacterial suspension. The standardized suspension of the bacteria 
Table Percentage of MSSA and MRSA Detected on the Samples

\begin{tabular}{|c|c|c|}
\hline Isolates & $n=65$ & Percentage $(\%)$ \\
\hline S. aureus + & 17 & 26.15 \\
\hline MSSA + & 11 & 16.92 \\
\hline MRSA + & 6 & 9.23 \\
\hline S. aureus - & 48 & 73.85 \\
\hline Total & 65 & 100 \\
\hline
\end{tabular}

was swabbed over the entire surface of the medium. Paper discs impregnated with $30 \mu \mathrm{g}$ cefoxitin were placed on the surface of the medium and the plate was incubated for 24 hours at $350 \mathrm{C}$ and diameter zones of inhibition (translucent area) around each disc were measured. A translucent resistance diameter $\leq 21 \mathrm{~mm}$ described a positive MRSA result and $\geq 22 \mathrm{~mm}$ described a positive methicillin sensitive staphylococcus aureus (MSSA) result.

The collected data were then plotted in tables and the proportions were calculated in percentage.

\section{Results}

This study was performed by selecting 65 samples of people aged 7-68 years who came to Puskesmas Jatinangor that met the inclusion criteria. This study revealed that one third of the samples were positive for S. aureus and approximately $9.23 \%$ from all samples were positive for MRSA (Table).

\section{Discussions}

This study discovered that susceptibility to CAMRSA was identified among people who met the inclusion criteria and came to Puskesmas Jatinangor, West Java. This study showed a significantly higher percentage compared to a study carried out in Indonesia. A study performed from July 2001 to May 2002 at two university hospitals in Indonesia ${ }^{3}$ included 3,995 inpatients, outpatients and relatives of patients, discovered about $9.1 \%$ of individuals are S. aureus nasal carriers, and after further testing with antibiotic resistance, it has found that $2 / 362$ of the S. aureus are MRSA.

The $\mathrm{S}$. aureus is a common commensal organism on human skin and mucosa. The anterior nares of the nose are the main ecological niche, while numerous other body sites may also harbor S. aureus including skin, pharynx, perineum and the gastrointestinal tract. Approximately $20-40 \%$ of healthy individuals carry $\mathrm{S}$. aureus in their anterior nares. Although a normal flora, it can cause significant infections ranging from local to deep seated infections. ${ }^{4}$

Staphylococcus aureus proliferates easily and causes infections whenever it is transferred to other areas of the body or if a favorable environment for growth is present. Resistance is often acquired by horizontal transfer to genes from outside sources, although chromosomal mutation and antibiotic selection are also important. ${ }^{5}$ As S. aureus easily adapts to and develops resistance to most antibiotics, hence it is very difficult to treat and will cause severe complications when treatment is delayed as it will cause sepsis when the bacteria enter systemic circulation. ${ }^{5}$

There are two types of MRSA, namely Hospital-Associated MRSA (HA-MRSA) and Community-Associated MRSA (CA-MRSA).5 Both types could manifest in hospital or community setting. ${ }^{6}$ The distinction of HAMRSA and CA-MRSA is that almost $100 \%$ of CA-MRSA has Panton and Valentine leukocidin (PVL) toxin gene which releases PV leukotoxin as the main pathogenesis for causing dermonecrotic lesions, also as an important factor in necrotizing skin infections. ${ }^{7}$

Nasal acquisition of CA-MRSA has been associated with a 10-fold increase in risk of developing skin and soft tissue infections (SSTIs). ${ }^{6}$ The symptoms of SSTIs are characterized by the usual red, swollen, painful, warm to the touch, full of pus or other drainage and accompanied by fever. These symptoms are commonly misleadingly referred to as spider or insect bites and treated with normal ointment. Hence if the appropriate treatment is delayed, bacteria might enter the bloodstream causing severe bloodstream infections (BSIs) which will then cause sepsis. A recent report showsed that $60 \%$ mortality for BSIs is caused by USA600 strain type. ${ }^{8}$

The CA-MRSA-associated skin and softtissue infections are treated with oral 
antibiotics including doxycycline, minocycline, clindamycin, trimethoprim-sulfamethoxazole, rifampicin and fusidic acid. Severe CA-MRSA infections and HA-MRSA require intravenous vancomycin therapy. Asymptomatic carriers represent an important MRSA reservoir. The transmission of MRSA infections may be limited by universal infection-control measures, patient education, screening and decolonization of asymptomatic MRSA carriers in both health-care and community settings. ${ }^{9}$

Education to the patient includes keeping the skin intact can protect against transmission, and by wearing gloves when performing activities likely cause punctures, scratches, cuts, abrasions or other injuries to the skin will help prevent CA-MRSA from causing skin and soft tissue infections. ${ }^{6}$ If injury to the skin occurs, the wound should be cleaned immediately with soap and warm water, dried, and then covered with a clean, dry bandage or dressing. Other strategies include hand washing with antimicrobial soap or alcohol based sanitizer; laundry (e.g. used clothing, towels, sheets and blankets) should be washed with detergent in water at $\geq 160^{\circ} \mathrm{F}$ for at least 25 minutes; use an automatic dryer on hot temperature $\left(180^{\circ} \mathrm{F}\right)$ to dry items-do not air dry and items should be used only after they are completely dry, besides that also to clean and disinfect contaminated items and environmental surfaces; Family, household members in close contacts to the carrier or patient should perform frequent hand washing with warm water and soap or alcohol-based hand sanitizers; Practice good personal hygiene including showering as soon as possible after physical activity, direct contact sports, or working out, make sure to use a clean and dry towel; Do not share personal care items (i.e., razors, towels, bar soap, and water bottles), any topical preparations (i.e., ointments, balm, lotions, deodorants, antibiotic creams) and shave body skin for cosmetic or wound care reasons, as shaving can create micro-abrasions that can permit entry of bacteria. ${ }^{6}$

Furthermore, routine screening for CAMRSA colonization in nares or other site is not recommended in the non-outbreak setting. 6 In selected circumstances, screening may be considered, for example if the individual has more than 2 times recurrent $\mathrm{S}$. aureus skin infections within 6 months, family settings where the recurrent skin infection still occurs despite review and re-inforcement of hygiene measures with known high prevalence of CAMRSA in the community and also to investigate an outbreak. ${ }^{6}$
Decolonization refers to eradication and reducing carriage of a certain organism. 6 It can be temporarily achieved but a significant portion of patients and health care workers become re-colonized within a relatively short time. The short-term mupirocin use may help interrupt nosocomial outbreaks, but infection control measures may be equally or more important. It is not recommended for usual management of individual with CA-MRSA infections, endemic infection or outbreak; should be considered only in exceptional circumstances which include cases if a patient develops a recurrent SSTI despite optimizing wound care and hygiene measures, ongoing transmission is occurring among household members or other close contacts despite optimizing wound care and hygiene measures. ${ }^{10}$

Decolonization strategies include nasal decolonization with mupirocin twice daily for 5-10 days; nasal decolonization with mupirocin twice daily for 5-10 days and topical body decolonization regimens with a skin antiseptic solution (e.g., chlorhexidine) for 5-14 days or dilute bleach baths. (For dilute bleach baths, 1 teaspoon per gallon of water [or $1 / 4$ cup per $1 / 4$ tub or 13 gallons of water] given for $15 \mathrm{~min}$ twice weekly for $\sim 3$ months can be considered.). ${ }^{10}$

As of limitations of this study, the lack of resource might play a definitive role as Polymerase Chain Reaction (PCR) for identification of mecA gene presence in the strain is the "golden standard". There were possibilities of false positive as the culture method for MRSA had a sensitivity of $83.3 \%$ and a specificity of $98.2 \% .14$ The sample used was not evenly distributed as it was not randomized with lack of samples collected due to costly culturing methods for detection of MRSA. In conclusion, The proportion of susceptibility to CA-MRSA carriers in people who came to Puskesmas Jatinangor is $9.23 \%$.

\section{References}

1. DeLeo FR, Otto M, Kreiswirth BN, Chambers HF. Community-associated meticillinresistant Staphylococcus aureus. Lancet. 2010;375(9725):1557-68.

2. Otter JA, French GL. Community-associated meticillin-resistant Staphylococcus aureus strains as a cause of healthcareassociated infection. J Hosp Infect. 2011;79(3):189-93.

3. Lestari ES, Severin JA, Filius PM, Kuntaman K, Duerink DO, Hadi U, et al. Antimicrobial 
resistance among commensal isolates of Escherichia coli and Staphylococcus aureus in the Indonesian population inside and outside hospitals. Eur J Clin Microbiol Infect Dis. 2008;27(1):45-51.

4. Ramana KV, Mohanty SK, Wilson CG. Staphylococcus aureus colonization of anterior nares of school going children. Indian J Pediatr. 2009;76(8):813-6.

5. Chambers HF, Deleo FR. Waves of resistance: Staphylococcus aureus in the antibiotic era. Nat Rev Microbiol. 2009;7(9):629-41.

6. Barton M, Hawkes M, Moore D, Conly J, Nicolle L, Allen U, et al. Guidelines for the prevention and management of community-associated methicillinresistant Staphylococcus aureus: A perspective for Canadian health care practitioners. Can J Microbiol. 2006;17(Suppl C):4C-24C.

7. Kuehnert MJ, Kruszon-Moran D, Hill HA,
McQuillan G, McAllister SK, Fosheim G, et al. Prevalence of Staphylococcus aureus nasal colonization in the United States, 20012002. J Infect Dis. 2006;193(2):172-9.

8. Donabedian SM, Moore CL, Perri MB, Chua T, Zervos MJ. Do laboratory characteristics predict outcome in methicillin-resistant staphylococcus aureus bacteremia? Clin Microbiol Infect. 2009;15(s4):1860.

9. Ray P, Gautam V, Singh R. Methicillinresistant staphylococcus aureus in developed and developing countries: Implications and solutions. Regional Health Forum (WHO/SEARO). 2011;15:74-82.

10. Liu C, Bayer A, Cosgrove SE, Daum RS, Fridkin SK, Gorwitz RJ, etal.Clinical practice guidelines by the infectious diseases society of america for the treatment of methicillin-resistant Staphylococcus aureus infections in adults and children. Clin Infect Dis. 2011;52(3):18-55. 